\title{
Alcohol and Cancer: Waiting for the Storm to Pass or Dancing in the Rains!
}

For an oncologist, cancer is about focusing on the fight and not the fright; however, for the patient and patient's family, it is frightening most of the times! There are many risk factors associated with cancer, including modifiable and nonmodifiable. Chronic alcohol abuse is one of the modifiable risk factors, inundated with health problems including cancer. The International Agency for Research on Cancer monographs classify alcohol as a Group 1 carcinogenic agent which means that alcohol is carcinogenic to humans. ${ }^{[1]}$ The evidence is primarily furnished by the epidemiological studies including casecontrol and cohort studies which report the estimate of effect (relative risk). There is a paucity of randomized controlled trials assessing both the carcinogenicity and causal association of alcohol and cancers. The Continuous Update Project World Cancer Research Fund/American Institute for Cancer Research reinforces that consumption of alcohol is associated with the increased risk of cancer and also provides causal association. ${ }^{[2,3]}$ There is an increased incidence for cancers of 7 subsites secondary to alcohol consumption. These are lip and oral cavity, oropharynx and hypopharynx, esophagus, colorectal, liver, larynx, and breast. ${ }^{[4]}$ The benefit of moderate drinking of alcohol is controversial with upsurgence of recent data demonstrating the contrary. ${ }^{[5,6]}$

Tobacco and alcohol are both preventable causes of cancers. The effect of simultaneous consumption of the two is not additive but synergistic. While the efforts are being made to curb the tobacco consumption at various levels, the measures are relatively less proactive when it comes to alcohol. There can be multiple reasons to it including lesser alcohol consumption in South East Asia compared to Western world, industry-driven motives, and, to an extent, lack of awareness.

\section{Carcinogenesis}

The carcinogenesis of alcohol is through multiple metabolic pathways which are not completely understood. ${ }^{[4,7]}$ Alcohol dehydrogenase is the enzyme that converts ethanol to acetaldehyde. Acetaldehyde with the help of enzyme aldehyde dehydrogenase is further metabolized to acetate. The main carcinogenic potential of alcohol is due to the metabolite acetaldehyde which causes DNA damage. Ethanol interferes with the metabolism of estrogen. The levels of this hormone are increased by alcohol consumption. Estrogen causes excessive cellular proliferation leading to breast cancer. There are various other mechanisms involved in the carcinogenesis of alcohol; one of them is by producing reactive oxygen species along the metabolic pathway. This oxidative stress leads to lipid peroxidation which causes the production electrophilic compounds resulting in the formation of exocyclic DNA adducts. Consumption of alcohol and tobacco has synergistic effect which can be explained by the fact that the alcohol acts as a solvent and facilitates absorption of carcinogens through the mucosa of the upper aerodigestive tract.

Genetic factors are also thought to play a role in the carcinogenesis of alcohol through polymorphisms of genes encoding enzymes and proteins involved in alcohol metabolism, folate and methionine metabolic pathways, and DNA repair. ${ }^{[8]}$ Mendelian randomization studies confirmed the above finding. The variant of aldehyde dehydrogenase ALDH2 $* 2$ is prevalent in the Asian population. The individual homozygous or heterozygous for this allele is unable to oxidize aldehyde or do it insufficiently leading to build up of acetaldehyde and has higher chances of developing colorectal and aerodigestive tract cancers. Methylenetetrahydrofolate reductase is the enzyme important for DNA synthesis and methylation. This enzyme converts 5,10-methylenetetrahydrofolate to 5-methylenetetrahydrofolate. The variant of this enzyme reduces its activity and its role has been noted with colon cancer.

\section{Consumption and Disease Burden}

The problem of alcohol consumption is more in the West compared to Indian subcontinent. Age-standardized prevalence of current drinking (patients consuming alcohol within the last 12 months) in Indian subcontinent for females is between $0 \%-19.9 \%$ compared to $40 \%-79.9 \%$ in the Western world and for males is $0 \%-39.9 \%$ compared to $40 \%-100 \%$ in the West. Age-standardized average standard drinks daily (defined as $10 \mathrm{~g}$ of pure ethanol per serving) among current drinkers in Indian subcontinent is reported as $1.01-2$ for males and $<1.0$ for females. ${ }^{[5]}$

Systematic analysis performed by global burden of Diseases, Injuries, and Risk Factors Study (GBD) 2016 alcohol collaborators generated an estimate of alcohol use and alcohol attributable deaths and disability-adjusted life years (DALYs) for 195 countries and territories spanning over two and a half decade. The results of the study reported that globally alcohol was the seventh leading risk factor for both deaths and DALYs in 2016. In 2016, there were 2.8 million alcohol-attributable deaths which corresponded to $2.2 \%$ of total age standardized deaths among females and $6.8 \%$ in males globally. It was the leading risk factor for death globally for the 
population aged 15-49 years, with alcohol-attributable death being $3.8 \%$ and $12.2 \%$ among female and male, respectively. Among the population aged 50 years or above, alcohol-induced cancer accounted for $27.1 \%$ and $18.9 \%$ of total alcohol-attributable deaths among females and males, respectively. Globally, in 2016, 1.6\% of total DALYs were lost due to alcohol in females and $6.0 \%$ among males in terms of overall disease burden. Alcohol use led to $8.9 \%$ of attributable DALYs lost for males and $2.3 \%$ for females among population aged $15-49$ years. ${ }^{[5]}$

\section{Zero Level of Alcohol Consumption is Safe to Minimize the Health Risk}

The study by GBD 2016 alcohol collaborators also showed that overall risk to health loss was minimized at consumption level of zero standard drinks daily. For alcohol-attributable disease outcomes including cancers, the risk rose monotonically on increasing alcohol consumption. For ischemic heart disease, the minimum relative risk was 0.86 for men and 0.82 for women with the consumption of $<1$ standard drink daily for both. The relative risk was similar across all ages. However, the protective effects of alcohol on ischemic heart disease and diabetes were offset by the risk associated with cancers which increased with the alcohol consumption. ${ }^{[5]}$

In 2016, alcohol caused $4.2 \%$ of all cancer deaths with the estimated number of 376,200 cancer deaths with an age standardized rate of 4.8 deaths per 100,000 people. Major proportion of these deaths were constituted by colorectal, liver, and esophagus in order of decreasing numbers. Alcohol consumption caused 10.3 million DALYs lost due to cancer in 2016 corresponding to $4.2 \%$ of all cancer DALYs lost. There is geographic variation for alcohol-attributable cancer burden, being lowest in North Africa and Middle East and highest in Europe. Age-standardized cancer deaths attributable to alcohol consumption in 2016 is between 0.8 and $3.3 / 100,000$ people representing up to $3.3 \%$ of cancer deaths and 24-103 age standardized cancer DALYs attributable to alcohol per $1,000,000$ people attributable to alcohol in Indian subcontinent. ${ }^{[2,9]}$

\section{Measures to Decrease Alcohol Consumption}

Alcohol is a preventable cause of cancer and hence efforts should be directed to decrease its consumption. Paradoxically, everything can be preserved in alcohol, but for health, wealth and happiness. A study by Alattas et al. looked at alcohol policies and alcohol-attributable cancer mortality. They reported that more restrictive alcohol policies were associated with lower cancer mortality. A $10 \%$ increase in the restrictiveness of the policies led to an $8.5 \%$ decrease in rates of combined alcohol attributable cancers. ${ }^{[10]}$ Schwartz et al. reported significant association between alcohol sales and alcohol mortality which persisted after controlling the tobacco use. Reducing alcohol consumption is a cost-effective means of targeting cancer control, which has the potential to decrease the cancer related mortality. ${ }^{[11]}$

Therefore, efforts should be directed towards individual, society, and legislative level to reduce the consumption of alcohol to decrease both the cancer incidence and the mortality. ${ }^{[12]}$

\section{Richa Vaish', Jyoti Bajpai}

${ }^{\prime}$ Department of Surgical Oncology, Tata Memorial Centre, Tata Memorial Hospital, Homi Bhabha National Institute, Mumbai, Maharashtra, India, ${ }^{2}$ Department of Medical Oncology, Tata Memorial Centre, Tata Memorial Hospital, Homi Bhabha National Institute, Mumbai, Maharashtra, India

Address for correspondence: Dr. Jyoti Bajpai, Department of Medical Oncology, Tata Memorial Centre, Tata Memorial Hospital, Homi Bhabha National Institute, Dr. Ernest Borges Marg, Parel, Mumbai - 400 012, Maharashtra, India. E-mail:drjyotibajpai25@gmail.com

Submitted: 09-Mar-2020

Revised: $30-A p r-2020$

Accepted: 01-May-2020

Published: 29-Aug-2020

\section{References}

1. IARC. Alcohol Consumption and Ethyl Carbamate IARC Monographs on the Evaluation of Carcinogenic Risks to Humans; 2010. Available from: https://publications.iarc.fr/96. [Last accessed on 2020 Feb 10].

2. Alcohol Consumption a Leading Risk Factor for Cancer. World Cancer Report. Cancer Research for Cancer Prevention. p. 68-77. Available from. https://www.iarc.fr/cards_page/ world-cancer-report/. [Last accessed on 2020 Feb 10].

3. WCRF/AICR. Diet, Nutrition, Physical Activity and cancer: A Global Perspective. Continuous Update Project Expert Report 2018. World Cancer Research Fund/American Institute for Cancer Research; 2018. Available from: https://www.wcrf.org/ dietandcancer. [Last accessed on $2020 \mathrm{Feb} 10]$.

4. Boffetta P, Hashibe M. Alcohol and cancer. Lancet Oncol 2006; 7:149-56.

5. GBD 2016 Alcohol Collaborators. Alcohol use and burden for 195 countries and territories, 1990-2016: A systematic analysis for the Global Burden of Disease Study 2016. Lancet 2018;392:1015-35.

6. Wood AM, Kaptoge S, Butterworth AS, Willeit P, Warnakula S, Bolton $\mathrm{T}$, et al. Risk thresholds for alcohol consumption: Combined analysis of individual-participant data for 599912 current drinkers in 83 prospective studies. Lancet 2018;391:1513-23.

7. Connor J. Alcohol consumption as a cause of cancer. Addiction 2017;112:222-8.

8. Druesne-Pecollo N, Tehard B, Mallet Y, Gerber M, Norat T, Hercberg S, et al. Alcohol and genetic polymorphisms: Effect on risk of alcohol-related cancer. Lancet Oncol 2009;10:173-80.

9. WHO. Global Status Report on Alcohol and Health 2018. Geneva, Switzerland: World Health Organization; 2018. Available from: https://www.who.int/substance_abuse/publications/global_ alcohol_report/en/.

10. Alattas M, Ross CS, Henehan ER, Naimi TS. Alcohol policies and alcohol-attributable cancer mortality in U.S. States. Chem Biol Interact 2020;315:108885.

11. Schwartz N, Nishri D, Chin Cheong S, Giesbrecht N, Klein-Geltink J. Is there an association between trends in alcohol 
consumption and cancer mortality? Findings from a multicountry analysis. Eur J Cancer Prev 2019;28:45-53.

12. Burton R, Henn C, Lavoie D, O'Connor R, Perkins C, Sweeney $\mathrm{K}$, et al. A rapid evidence review of the effectiveness and cost-effectiveness of alcohol control policies: An English perspective. Lancet 2017;389:1558-80.
This is an open access journal, and articles are distributed under the terms of the Creative Commons Attribution-NonCommercial-ShareAlike 4.0 License, which allows others to remix, tweak, and build upon the work non-commercially, as long as appropriate credit is given and the new creations are licensed under the identical terms.

\begin{tabular}{|l|l|}
\hline \multicolumn{2}{|c|}{ Access this article online } \\
\hline Quick Response Code: & Website: \\
& www.ijmpo.org \\
\cline { 2 - 2 } & DOI: \\
\hline
\end{tabular}

How to cite this article: Vaish R, Bajpai J. Alcohol and cancer: Waiting for the storm to pass or dancing in the rains! Indian J Med Paediatr Oncol 2020;41:473-5. 